\title{
ON DIFFERENTIABILITY WITH RESPECT TO THE INITIAL DATA OF A SOLUTION OF AN SDE WITH LÉVY NOISE AND DISCONTINUOUS COEFFICIENTS
}

\author{
OLGA V. ARYASOVA AND ANDREY YU. PILIPENKO
}

\begin{abstract}
We construct a stochastic flow generated by an SDE with its drift being a function of bounded variation and its noise being a stable process with exponent from $(1,2)$. It is proved that the flow is non-coalescing and Sobolev differentiable with respect to initial data. The representation for the derivative is given.
\end{abstract}

\section{INTRODUCTION}

Consider an SDE

$$
\varphi_{t}(x)=x+\int_{0}^{t} a\left(\varphi_{s}(x)\right) d s+Z(t), t \geq 0
$$

where $x \in \mathbb{R}, a$ is a bounded measurable function on $\mathbb{R},(Z(t))_{t \geq 0}$ is a symmetric stable process with the exponent $\alpha \in(1,2)$, i.e. $(Z(t))_{t \geq 0}$ is a Lévy process with its characteristic function being equal to

$$
E \exp \{i \lambda Z(t)\}=\exp \left\{-c t|\lambda|^{\alpha}\right\}, \lambda \in \mathbb{R},
$$

where $c>0$ is a constant.

The existence and uniqueness of a weak solution, and the strong Markov property was considered in [7, 13]. The existence of a unique string solution for equation (1) was proved in [9. Besides, it is continuously dependent on $x$ :

$$
\forall T>0 \forall x_{0} \in \mathbb{R}: \sup _{t \in[0, T]}\left|\varphi_{t}(x)-\varphi_{t}\left(x_{0}\right)\right| \stackrel{P}{\rightarrow} 0, x \rightarrow x_{0} .
$$

In this paper we construct a modification of $\left(\varphi_{t}(x)\right)_{t \geq 0}$ which is cádlág in $t$ and monotonous in $x$. We prove that if a function $a$ has a locally bounded variation, then $\left(\varphi_{t}(x)\right)_{t \geq 0}$ is Sobolev differentiable in $x$ a.s. and the derivative $\nabla \varphi_{t}(x):=\frac{\partial \varphi_{t}(x)}{\partial x}$ has the following representation

$$
\nabla \varphi_{t}(x)=\exp \left\{\int_{\mathbb{R}} L_{t}^{\varphi(x)}(y) d a(y)\right\}
$$

where $L_{t}^{\varphi(x)}(y)$ is a local time of the process $\left(\varphi_{s}(x)\right)_{s \in[0, t]}$ at the point $y$.

Formula (2) can be easily explained for $a \in C^{1}(\mathbb{R})$. Indeed, in this case for each $\omega$, equation (11) can be considered as an integral equation with continuously differentiable coefficients. Then $\varphi_{t}(x)$ is continuously differentiable in $x$ and $\nabla \varphi_{t}(x)$ satisfies the linear equation

$$
\nabla \varphi_{t}(x)=1+\int_{0}^{t} a^{\prime}\left(\varphi_{s}(x)\right) \nabla \varphi_{s}(x) d s
$$

So

$$
\nabla \varphi_{t}(x)=\exp \left\{\int_{0}^{t} a^{\prime}\left(\varphi_{s}(x)\right) d s\right\}
$$

2000 Mathematics Subject Classification. 60J65, 60H10.

Key words and phrases. stochastic flow, stable process, local time, differentiability with respect to initial data. 
By the occupation times formula [3], the r.h.s. of (4) is equal to

$$
\exp \left\{\int_{\mathbb{R}} a^{\prime}(y) L_{t}^{\varphi(x)}(y) d y\right\}=\exp \left\{\int_{\mathbb{R}} L_{t}^{\varphi(x)}(y) d a(y)\right\} \text { a.s. }
$$

Remark 1. All the technical details needed for the existence of local time such as the validity of occupation times formula, the existence of the integrals etc. will be given in the next sections.

To prove (2) for $a$ being a function of bounded variation we will use an approximation of (11) by SDEs with $C^{1}$ drifts.

If $(Z(t))_{t>0}$ is a Wiener process that corresponds to $\alpha=2$ then the similar problem is well studied even for non-additive noises (see for example [2, 4, 5, 8). Note that most techniques used in a Wiener case for non-smooth a (Zvonkin's transformation, Tanaka's formula, Girsanov's formula etc.) are inapplicable to a case of Lévy process. It is worth note that the differentiability w.r.t. initial data of solutions of SDEs with jumps and non-smooth coefficients has not been studied.

The paper is organized as follows. The results on measurability and continuity of the solution, and the estimates on transition density are represented in Section 2, In section 3 we give a definition of a local time and prove the existence of the local time for the process $\left(\varphi_{t}(x)\right)_{t \geq 0}$. The main result on differentiability of the solution is given in Section 4. Theorem 2.

\section{Properties of SOlution}

In this section we construct a version of $\left(\varphi_{t}(x)\right)_{t \geq 0}$ satisfying some measurability properties.

Put $\mathcal{F}_{t}=\sigma\{Z(s): 0 \leq s \leq t\}$.

Proposition 1. Let $a(x), x \in \mathbb{R}$, be a bounded measurable function. Then

1) There exists a unique strong solution of (11), i.e. a $\mathcal{F}_{t}$-adapted cádlág process $\left(\varphi_{t}(x)\right)_{t \geq 0}$ that satisfies (10) almost surely.

2) The process $\left(\varphi_{t}(x)\right)_{t \geq 0}$ is continuous w.r.t. $x$ in probability in topology of uniform convergence:

$$
\forall T>0 \forall x_{0} \in \mathbb{R}: \quad \sup _{t \in[0, T]}\left|\varphi_{t}(x)-\varphi_{t}\left(x_{0}\right)\right| \stackrel{P}{\rightarrow} 0, x \rightarrow x_{0} .
$$

3) The process $\left(\varphi_{t}(x)\right)_{t \geq 0}$ is a homogeneous strong Markov process. It has a continuous transition density $p_{t}(x, y)$. Moreover,

$$
\forall T>0 \exists N_{T}=N_{T,\|a\|_{L_{p}}} \forall t \in(0, T] \forall x \in \mathbb{R}, y \in \mathbb{R}:
$$

$$
p_{t}(x, y) \leq \frac{N_{T} t}{\left(t^{1 / \alpha}+|y-x|\right)^{\alpha+1}},
$$

where $\alpha \in(1,2)$ is a parameter of a stable process $(Z(t))_{t \geq 0}$.

4) If $x_{1} \leq x_{2}$, then

$$
P\left\{\varphi_{t}\left(x_{1}\right)=\varphi_{t}\left(x_{2}\right), t \geq \sigma_{x_{1}, x_{2}}\right\}=1,
$$

where

$$
\sigma_{x_{1}, x_{2}}=\inf \left\{t \geq 0: \varphi_{t}\left(x_{1}\right) \geq \varphi_{t}\left(x_{2}\right)\right\} .
$$

5) The process $\left(\varphi_{t}(x)\right)_{t \geq 0, x \in \mathbb{R}}$ can be selected such that

a) it is monotonous in $x$ :

$$
\forall \omega \in \Omega \forall x_{1} \leq x_{2} \forall t \geq 0: \varphi_{t}\left(x_{1}, \omega\right) \leq \varphi_{t}\left(x_{2}, \omega\right) ;
$$

b) it is cádlág in $x$ for any fixed $t$ and $\omega$;

c) for any $T>0$ a map

$$
[0, T] \times \mathbb{R} \times \Omega \ni(t, x, \omega) \mapsto \varphi_{t}(x, \omega)
$$

is $\mathcal{B}([0, T]) \times \mathcal{B}(\mathbb{R}) \times \mathcal{F}_{T}$-measurable. 
Proof. For a proof of 1), 2), see [9, 3) even in more general case is proved in [10, 13].

Prove 4). Since the process

$$
\varphi_{t}\left(x_{2}\right)-\varphi_{t}\left(x_{1}\right)=\int_{0}^{t}\left(a\left(\varphi_{s}\left(x_{2}\right)\right)-a\left(\varphi_{s}\left(x_{1}\right)\right)\right) d s, t \geq 0,
$$

is continuous in $t$, then

$$
\sigma_{x_{1}, x_{2}}=\inf \left\{t \geq 0: \varphi_{t}\left(x_{1}\right)=\varphi_{t}\left(x_{2}\right)\right\},
$$

and it is easy to see that the process

$$
\widetilde{\varphi}_{t}\left(x_{1}\right):=\left\{\begin{array}{l}
\varphi_{t}\left(x_{1}\right), t \leq \sigma_{x_{1}, x_{2}}, \\
\varphi_{t}\left(x_{2}\right), t>\sigma_{x_{1}, x_{2}}
\end{array}\right.
$$

is a solution of (11) with initial value $x_{1}$. By the uniqueness of the solution this implies (77).

We construct a version of the solution that satisfies properties of the last part of Proposition 1. Let $\widetilde{\Omega}$ be a set of full measure such that (10) and (8) are satisfied for all $\omega \in \widetilde{\Omega}, t \geq 0$, and rational $x$. The monotonicity and (5) imply that $\widetilde{\Omega}$ can be selected such that

$$
\begin{aligned}
\forall \omega \in \widetilde{\Omega} \forall x_{0} \in \mathbb{Q} & \forall T>0: \\
& \sup _{t \in[0, T]}\left|\varphi_{t}(x, \omega)-\varphi_{t}\left(x_{0}, \omega\right)\right| \rightarrow 0, x \rightarrow x_{0}, x \in \mathbb{Q} .
\end{aligned}
$$

It is easy to see that

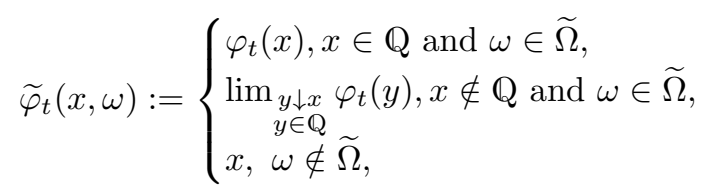

is a version of $\left(\varphi_{t}(x)\right)_{t \geq 0, x \in \mathbb{R}}$ that satisfies 5$)$.

The Proposition is proved.

Remark 2. Later on we will always consider a version of $\left(\varphi_{t}(x)\right)_{t \geq 0}$ that satisfies the assumptions of Proposition 1 .

\section{A local time}

In this section we prove the existence and study properties of a local time for the process $\left(\varphi_{t}(x)\right)_{t \geq 0}$.

There are a few different approaches to the notion of a local time. We consider a local time as a density of the occupation measure. Recall the definition and some properties of local times (see [3, 6]).

Let $X(t), t \geq 0$, be a measurable function. Define a measure

$$
\nu_{t}(A):=\lambda\{s: X(s) \in A, s \in[0, t]\},
$$

where $\lambda$ is a Lebesgue measure.

Definition 1. Let the measure $\nu_{t}$ be absolutely continuous w.r.t. $\lambda$. Then its RadonNikodym derivative $\alpha(y, t)=\frac{d \nu_{t}(y)}{d \lambda}$ is called a local time of $X$ relative to $[0, t]$. In particular,

$$
\begin{aligned}
\nu_{t}(A) & =\int_{A} \alpha_{t}(y) d y, A \in \mathcal{B}(\mathbb{R}), \\
\int_{0}^{t} f(X(s)) d s & =\int_{\mathbb{R}} f(y) \nu_{t}(d y)=\int_{\mathbb{R}} f(y) \alpha_{t}(y) d y,
\end{aligned}
$$

where $f$ is a measurable function for which at least one integral in (9) make sense. 
Definition 2. Let $X(t), t \geq 0$, be a measurable stochastic process. We say that the local time of $X$ exists a.s. if almost all trajectories have a local time.

Assume that for any $0<t_{1}<t_{2}<\ldots<t_{n}$ the distribution of $\left(X\left(t_{1}\right), \ldots, X\left(t_{n}\right)\right)$ is absolutely continuous. Let $p\left(x_{1}, \ldots, x_{n}, t_{1}, \ldots, t_{n}\right)$ be the corresponding density.

Put

$$
q_{t}\left(x_{1}, \ldots, x_{n}\right)=\int_{0}^{t} \ldots \int_{0}^{t} p\left(x_{1}, \ldots, x_{n}, t_{1}, \ldots, t_{n}\right) d t_{1} \ldots d t_{n} .
$$

Theorem 1 (See [3]). If for some $n \geq 2$ the function $q_{t}$ is continuous, then the local time exists a.s., and

$$
E \alpha_{t}\left(x_{1}\right) \cdot \ldots \cdot \alpha_{t}\left(x_{n}\right)=q_{t}\left(x_{1}, \ldots, x_{n}\right) .
$$

Remark 3. It was mentioned in the proof (see also [6], Sect.25) that

$$
(2 \varepsilon)^{-1} \int_{0}^{t} \mathbb{1}_{\{|X(s)-y|<\varepsilon\}} d s \rightarrow \alpha_{t}(y), \varepsilon \rightarrow 0+,
$$

in $L_{2}$-sense for any $y \in \mathbb{R}$, and almost surely for $\lambda$-a.a. $y$. It follows from (11) and standard results on existence of measurable version of a limit (see, for example, [14]), that the local time can be selected measurable in $(y, \omega)$. Further we consider only such a modification.

Remark 4. Note that if the local time exists a.s., then (9) is satisfied with probability one for any measurable non-negative function $f$. The exceptional set is independent of $f$.

Return to equation (11).

Proposition 2. There exists a process $\alpha_{x}(y, t), x \in \mathbb{R}, y \in \mathbb{R}, t \geq 0$, such that

1) for any fixed $x: \alpha_{x}(y, t)$ is a local time of $\varphi_{s}(x), s \in[0, t]$;

2) $\alpha_{x}(y, t)$ is measurable in $(x, y, \omega, t)$;

3) for any $x \in \mathbb{R}, t>0$, a map $y \mapsto \alpha_{x}(y, t)$ is continuous in $L_{2}$.

Proof. The existence of the local time for fixed $x$ follows from Theorem 1 and Proposition 1 Indeed, let $n=2$, then

$$
\begin{gathered}
p\left(x_{1}, x_{2}, t_{1}, t_{2}\right)=p_{t_{1}}\left(x, x_{1}\right) p_{t_{2}-t_{1}}\left(x_{1}, x_{2}\right) \leq \\
\leq\left(N_{t}\right)^{2} t_{1}^{1-\frac{\alpha+1}{\alpha}}\left(t_{2}-t_{1}\right)^{1-\frac{\alpha+1}{\alpha}}= \\
=\left(N_{t}\right)^{2} t_{1}^{-\frac{1}{\alpha}}\left(t_{2}-t_{1}\right)^{-\frac{1}{\alpha}}, 0<t_{1}<t_{2} \leq t .
\end{gathered}
$$

Observe that

$$
\begin{aligned}
& \int_{0}^{t} \int_{0}^{t_{2}}\left(N_{t}\right)^{2} t_{1}^{-\frac{1}{\alpha}}\left(t_{2}-t_{1}\right)^{-\frac{1}{\alpha}} d t_{1} d t_{2}= \\
= & \left(N_{t}\right)^{2} \int_{0}^{t} \int_{0}^{1}\left(z t_{2}\right)^{-\frac{1}{\alpha}}\left(t_{2}-z t_{2}\right)^{-\frac{1}{\alpha}} t_{2} d z d t_{2}= \\
= & \left(N_{t}\right)^{2} \int_{0}^{t} t_{2}^{1-\frac{2}{\alpha}} B\left(1-\frac{1}{\alpha}, 1-\frac{1}{\alpha}\right) d t_{2}<\infty .
\end{aligned}
$$

Above we have used that $0<1-\frac{1}{\alpha}$ and $1-\frac{2}{\alpha}>-1$ because $\alpha \in(1,2)$.

The continuity of $q_{t}$ follows from the Lebesgue dominated convergence theorem.

Remark 3 allows us to select a measurable in $(x, y, \omega, t)$ modification.

The Proposition is proved.

Remark 5 . The local time from Proposition 2 coincides with that obtained by N.I.Portenko [1], who considered it as a $W$-functional from Markov process.

In the following statement we obtain the exponential integrability of the local time. 
Proposition 3. For any $t>0, \mu>0$, there exists $c=c\left(t,\|a\|_{\infty}, \mu\right)$ such that

$$
\forall x, y \in \mathbb{R} \quad E \exp \left\{\mu \alpha_{x}(y, t)\right\} \leq c .
$$

A possible way to prove (13) is to expand the exponent in a Taylor series, then to use estimate (6), and to make calculations similar to (12) and formula (10). However it is easier to apply the following result of N.I.Portenko.

Lemma 1. Assume that $\{\beta(t), t \in[0, T]\}$ is a non-negative measurable process adapted to a flow $\left\{\mathcal{F}_{t}, t \in[0, T]\right\}$. Assume that for $0 \leq s \leq t \leq T$

$$
E\left\{\int_{s}^{t} \beta(\tau) d \tau / \mathcal{F}_{s}\right\} \leq \rho(s, t)
$$

where $\rho(s, t)$ is a non-random integral function satisfying the following conditions

a) $\rho\left(t_{1}, t_{2}\right) \leq \rho\left(t_{3}, t_{4}\right)$ if $\left(t_{1}, t_{2}\right) \subset\left(t_{3}, t_{4}\right)$;

b) $\lim _{h \downarrow 0} \sup _{0 \leq s \leq t \leq h} \rho(s, t)=0$.

Then for any $\bar{\lambda}$

$$
E \exp \left\{\lambda \int_{0}^{T} \beta(\tau) d \tau\right\} \leq c
$$

where $c$ depends only on $\lambda, T$ and $\rho$.

See [12, Lemma 1.1 for the proof.

Proof of Proposition 3. Let now $\beta_{\varepsilon}(t)=(2 \varepsilon)^{-1} \mathbb{1}_{\left|\varphi_{t}(x)-y\right|<\varepsilon}$. Similarly to (12) we obtain that uniformly in $x, y, \varepsilon$

$$
\begin{gathered}
E\left(\int_{s}^{t}(2 \varepsilon)^{-1} \mathbb{1}_{\left|\varphi_{\tau}(x)-y\right|<\varepsilon} d \tau / \mathcal{F}_{s}\right)= \\
=\int_{0}^{t-s} \int_{y-\varepsilon}^{y+\varepsilon}(2 \varepsilon)^{-1} p_{r}\left(\varphi_{s}(x), u\right) d u d r \leq \\
\leq \int_{0}^{t-s} \frac{N_{T} r}{r^{\frac{\alpha+1}{\alpha}}} d r=N_{T}(t-s)^{\frac{\alpha-1}{\alpha}} \cdot\left(\frac{\alpha}{\alpha-1}\right)=: \rho(s, t), 0 \leq s \leq t \leq T .
\end{gathered}
$$

This implies the uniform in $x, y, \varepsilon$ estimate of

$$
E \exp \left\{\lambda \int_{0}^{t}(2 \varepsilon)^{-1} \mathbb{1}_{\left|\varphi_{s}(x)-y\right|<\varepsilon} d s\right\} .
$$

To conclude the proof, it remains to make $\varepsilon \rightarrow 0$ and apply Fatou's lemma.

Proposition 3 is proved.

\section{Representation of the Derivative}

Consider equation (1). Assume that $a$ is continuously differentiable. Then for each $\omega \in \Omega$, (11) can be considered as an integral equation with $C^{1}$-coefficients. So $\varphi_{t}(x)$ is differentiable in $x$ and $\nabla \varphi_{t}(x)=\frac{\partial \varphi_{t}(x)}{\partial x}$ satisfies a linear equation

$$
\nabla \varphi_{t}(x)=1+\int_{0}^{t} a^{\prime}\left(\varphi_{s}(x)\right) \nabla \varphi_{s}(x) d s .
$$

Thus

$$
\nabla \varphi_{t}(x)=\exp \left\{\int_{0}^{t} a^{\prime}\left(\varphi_{s}(x)\right) d s\right\} .
$$

Applying (9) and Proposition 2 we get

$$
\nabla \varphi_{t}(x)=\exp \left\{\int_{\mathbb{R}} a^{\prime}(y) \alpha_{x}(y, t) d y\right\}=\exp \left\{\int_{\mathbb{R}} \alpha_{x}(y, t) d a(y)\right\} \text { a.s. }
$$


Note that generally speaking the exceptional set depends on $x$ and $t$. By Fubini's theorem

$$
P\left\{\nabla \varphi_{t}(x)=\exp \left\{\int_{\mathbb{R}} \alpha_{x}(y, t) d a(y)\right\} \text { for } \lambda \text {-a.a. } x\right\}=1 .
$$

We will justify representation (14) for solution of (1), where a function $a$ is not necessarily $C^{1}$, but it has a finite variation. We need some definitions and facts on Sobolev spaces.

Definition 3. A function $f:[a, b] \rightarrow \mathbb{R}$ belongs to a Sobolev space $W_{p}^{1}([a, b]), p \geq 1$, if $f$ has an absolutely continuous modification and $\frac{d f}{d x} \in L_{p}([a, b])$.

Put

$$
\|f\|_{p, 1}:=\|f\|_{W_{p}^{1}([a, b])}:=\|f\|_{L_{p}([a, b])}+\left\|\frac{d f}{d x}\right\|_{L_{p}([a, b])} .
$$

It is well known that $\left(W_{p}^{1}([a, b]),\|\cdot\|_{p, 1}\right)$ is a Banach space. So if $\left\{f_{n}\right\} \subset W_{p}^{1}([a, b])$ is such that

$$
f_{n} \rightarrow f, \text { and } \frac{d f_{n}}{d x} \rightarrow g \text { as } n \rightarrow \infty \text { in } L_{p}
$$

then

$$
f \in W_{p}^{1}([a, b]), g=\frac{d f}{d x} .
$$

Definition 4. A measurable function $f: \mathbb{R} \rightarrow \mathbb{R}$ belongs to the space $W_{p, l o c}^{1}$ if its restriction to any segment $[a, b]$ lies in $W_{p}^{1}([a, b])$.

The main result of the paper is the following theorem.

Theorem 2. Assume that $a(x), x \in \mathbb{R}$, is a measurable bounded function and its restriction to any interval has a finite variation. Then $\varphi_{t}(\cdot) \in W_{p, l o c}^{1}$ for any $p \geq 1$ a.s. and representation (14) holds true for any $t>0$.

Corollary 1. For all $\left\{x_{1}, x_{2}\right\} \subset \mathbb{R}, x_{1} \neq x_{2}$,

$$
P\left\{\varphi_{t}\left(x_{1}\right) \neq \varphi_{t}\left(x_{2}\right), t \geq 0\right\}=1 .
$$

Relation (17) can be obtained similarly to that of [1, Theorem 1, using the fact that

$$
\varphi_{t}\left(x_{2}\right)-\varphi_{t}\left(x_{1}\right)=\int_{x_{1}}^{x_{2}} \frac{\partial \varphi_{t}(y)}{\partial y} d y>0 .
$$

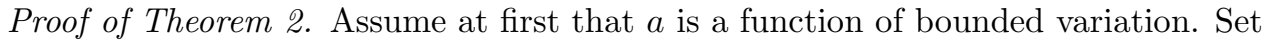

$$
a_{n}(x):=\int_{\mathbb{R}} a(y) g_{n}(x-y) d y,
$$

where

$$
g_{n}(x)=n g(n x), g \in C_{0}^{\infty}(\mathbb{R}), g \geq 0, \text { and } \int_{\mathbb{R}} g(z) d z=1 .
$$

Then

$$
\sup _{n, x}\left|a_{n}(x)\right| \leq\|a\|_{\infty}=\sup _{x}|a(x)|,
$$

$a_{n} \in C^{\infty}(\mathbb{R}), a_{n}(x) \rightarrow a(x), n \rightarrow \infty$, for all points of continuity of $a$, and

$$
\operatorname{Var}\left(a_{n}\right) \leq \operatorname{Var}(a)
$$

Let $\varphi_{t}^{n}(x)$ be a solution of (1) with $a_{n}$ instead of $a, \alpha_{x}^{n}(y, t)$ be its local time. Then

$$
P\left\{\nabla \varphi_{t}^{n}(x)=\exp \left\{\int_{\mathbb{R}} \alpha_{x}^{n}(y, t) d a_{n}(y)\right\} \text { for } \lambda \text {-a.a } x\right\}=1 .
$$

It follows from $[9$ that

$$
\forall x \forall T \geq 0: \sup _{t \in[0, T]}\left|\varphi_{t}^{n}(x)-\varphi_{t}(x)\right| \stackrel{P}{\rightarrow} 0, n \rightarrow \infty .
$$


The uniform boundedness of $\left\{a_{n}\right\}$ implies

$$
\forall p \geq 1: \sup _{x} \sup _{t \in[0, T]} E\left|\varphi_{t}^{n}(x)-\varphi_{t}(x)\right|^{p}<\infty .
$$

So

$$
E \int_{a}^{b}\left|\varphi_{t}^{n}(x)-\varphi_{t}(x)\right|^{p} d x \rightarrow 0, n \rightarrow \infty
$$

for any $t>0, a \leq b$.

Prove that

$\forall p \geq 0 \forall a \leq b \forall t>0$ :

$$
E \int_{a}^{b}\left|\exp \left\{\int_{\mathbb{R}} \alpha_{x}^{n}(y, t) d a_{n}(y)\right\}-\exp \left\{\int_{\mathbb{R}} \alpha_{x}(y, t) d a(y)\right\}\right|^{p} d x \rightarrow 0, n \rightarrow \infty,
$$

By Proposition 3. (18), and Jensen's inequality, it suffices to check the convergence

$$
\forall x, t: \int_{\mathbb{R}} \alpha_{x}^{n}(y, t) d a_{n}(y) \rightarrow \int_{\mathbb{R}} \alpha_{x}(y, t) d a(y), n \rightarrow \infty,
$$

in probability or in $L_{2}$-sense.

Assume at first that the function $a$ has a finite support. Let $R$ be such that supp $a \subset$ $[-R, R]$, supp $a_{n} \subset[-R, R]$.

For simplicity denote $a$ by $a_{0}$ and $\alpha$ by $\alpha^{0}$.

Let $-R=y_{0}<y_{1}<\ldots<y_{m}=R$ be a dissection of $[-R, R]$. Then

$$
\begin{aligned}
& E\left|\int_{\mathbb{R}} \alpha_{x}^{n}(y, t) d a_{n}(y)-\int_{\mathbb{R}} \alpha_{x}(y, t) d a(y)\right|=E\left|\int_{-R}^{R} \ldots-\int_{-R}^{R} \ldots\right| \leq \\
& \quad \leq \sum_{0 \leq j<m} E\left|\int_{y_{j}}^{y_{j+1}}\left(\alpha_{x}^{n}(y, t)-\frac{\int_{y_{j}}^{y_{j+1}} \alpha_{x}^{n}(z, t) d z}{\Delta y_{j}}\right) d a_{n}(y)\right|+ \\
& \quad+\sum_{0 \leq j<m} E\left|\int_{y_{j}}^{y_{j+1}}\left(\frac{\int_{y_{j}}^{y_{j+1}}\left(\alpha_{x}^{n}(z, t)-\alpha_{x}(z, t)\right) d z}{\Delta y_{j}}\right) d a_{n}(y)\right|+ \\
& \quad+\sum_{0 \leq j<m} E\left|\int_{y_{j}}^{y_{j+1}}\left(\frac{\int_{y_{j}}^{y_{j+1}} \alpha_{x}(z, t) d z}{\Delta y_{j}}\right)\left(d a_{n}(y)-d a(y)\right)\right|+ \\
& \quad+\sum_{0 \leq j<m} E\left|\int_{y_{j}}^{y_{j+1}}\left(\frac{\int_{y_{j}}^{y_{j+1}} \alpha_{x}(z, t) d z}{\Delta y_{j}}-\alpha_{x}(y, t)\right) d a(y)\right| \leq \\
& \quad \leq 2 \sup _{l \geq 0} \max _{0 \leq j<m} \sup _{y \in\left[y_{j}, y_{j+1}\right]} E\left|\alpha_{x}^{l}(y, t)-\frac{\int_{y_{j}}^{y_{j+1}} \alpha_{x}^{l}(z, t) d z}{\Delta y_{j}}\right| \operatorname{Var} a_{l}+ \\
& +\max _{0 \leq j<m} E\left|\int_{y_{j}}^{y_{j+1}} \alpha_{x}^{n}(z, t) d z-\int_{y_{j}}^{y_{j+1}} \alpha_{x}(z, t) d z\right| \frac{\operatorname{Var} a_{n}}{\min _{0 \leq j<m-1} \Delta y_{j}}+ \\
& \quad+\max _{0 \leq j<m} E \frac{\int_{y_{j}}^{y_{j+1}} \alpha_{x}(z, t) d z}{\Delta y_{j}} \sum_{k=0}^{m-1}\left|\Delta_{k} a_{n}-\Delta a_{k}\right|=I_{1}+I_{2}+I_{3},
\end{aligned}
$$

where $\Delta y_{j}=y_{j+1}-y_{j} ; \Delta_{k} a_{n}=\left(a_{n}\left(y_{k+1}\right)-a_{n}\left(y_{k}\right)\right)$.

Estimate each term in the r.h.s. of (19).

By (10) and (6), for any fixed $t \geq 0, x \in \mathbb{R}$, the processes $\alpha_{x}^{l}(y, t), y \in[-R, R]$, are equicontinuous in $L_{2}$ (and so in $L_{1}$ ) uniformly in $l \geq 0$, i.e.

$\forall \varepsilon>0 \forall l \geq 0 \exists \delta_{1}=\delta_{1}(\varepsilon)>0 \forall\left\{y^{\prime}, y^{\prime \prime}\right\} \subset[-R, R],\left|y^{\prime}-y^{\prime \prime}\right|<\delta:$

$$
\sqrt{E\left(\alpha_{x}^{l}\left(t, y^{\prime}\right)-\alpha_{x}^{l}\left(t, y^{\prime \prime}\right)\right)^{2}}<\varepsilon \text {. }
$$


Hence, if $\max _{0 \leq j<m}\left|y_{j+1}-y_{j}\right|<\delta_{1}$, where $\delta_{1}=\delta_{1}\left(\varepsilon /\left(6 \sup _{l>0} \operatorname{Var} a_{l}\right)\right)$ then the term $I_{1}$ in (19) is less than $\frac{\varepsilon}{3}$.

Consider $I_{2}$. By the definition of the local time (see (9)):

$$
\int_{y_{j}}^{y_{j+1}} \alpha_{x}^{n}(z, t) d z=\int_{0}^{t} \mathbb{1}_{\varphi_{z}^{n}(x) \in\left[y_{j}, y_{j+1}\right]} d z \text { a.s. }
$$

Therefore,

$$
\begin{aligned}
& E\left|\int_{y_{j}}^{y_{j+1}} \alpha_{x}^{n}(z, t) d z-\int_{y_{j}}^{y_{j+1}} \alpha_{x}(z, t) d z\right| \\
& \leq E \int_{0}^{t}\left|\mathbb{1}_{\varphi_{z}^{n}(x) \in\left[y_{j}, y_{j+1}\right]}-\mathbb{1}_{\varphi_{z}(x) \in\left[y_{j}, y_{j+1}\right]}\right| d z .
\end{aligned}
$$

Taking into account that by [9],

$$
\sup _{z \in[0, t]}\left|\varphi_{z}^{n}(x)-\varphi_{z}(x)\right| \stackrel{P}{\rightarrow} 0, n \rightarrow \infty,
$$

we get

$$
\mathbb{1}_{\varphi_{z}(x) \notin\left\{y_{j}, y_{j+1}\right\}}\left(\mathbb{1}_{\varphi_{z}^{n}(x) \in\left[y_{j}, y_{j+1}\right]}-\mathbb{1}_{\varphi_{z}(x) \in\left[y_{j}, y_{j+1}\right]}\right) \stackrel{P}{\rightarrow} 0, n \rightarrow \infty,
$$

for any $z$.

Since

$$
E \int_{0}^{t} \mathbb{1}_{\varphi_{z}(x) \in\left\{y_{j}, y_{j+1}\right\}} d z=\int_{\left\{y_{j}\right\} \cup\left\{y_{j+1}\right\}} E \alpha_{x}(z, t) d z=0,
$$

we have the convergence

$$
\left(\mathbb{1}_{\varphi_{z}^{n}(x) \in\left[y_{j}, y_{j+1}\right]}-\mathbb{1}_{\varphi_{z}(x) \in\left[y_{j}, y_{j+1}\right]}\right) \stackrel{P}{\rightarrow} 0, n \rightarrow \infty,
$$

and consequently the convergence

$$
E\left|\int_{y_{j}}^{y_{j+1}} \alpha_{x}^{n}(z, t) d z-\int_{y_{j}}^{y_{j+1}} \alpha_{x}(z, t) d z\right| \rightarrow 0, n \rightarrow \infty .
$$

Recall that

$$
a_{n}(y) \rightarrow a_{0}(y), n \rightarrow \infty,
$$

if $y$ is a point of continuity of $a_{0}$. Select a dissection $\left\{y_{k}\right\}$ such that all $\left\{y_{k}\right\}$ are points of continuity of $a_{0}$, and $\max _{j} \Delta y_{j}<\delta_{1}$. Use (20) and (21) and select $n_{0}$ such that for any $n \geq n_{0}:$

$$
\frac{\sup _{p \geq 0} \operatorname{Var} a_{p}}{\min _{0 \leq j<m} \Delta y_{j}} \cdot \max _{0 \leq j<m} E\left|\int_{y_{j}}^{y_{j+1}} \alpha_{x}^{n}(z, t) d z-\int_{y_{j}}^{y_{j+1}} \alpha_{x}(z, t) d z\right|<\frac{\varepsilon}{3}
$$

and

$$
\sup _{-R \leq z \leq R} E \alpha_{x}(z, t) \cdot \sum_{k=0}^{m-1}\left|\Delta_{k} a_{n}-\Delta_{k} a_{0}\right|<\frac{\varepsilon}{3}
$$

So the r.h.s. of (19) is less than $\varepsilon$ and the theorem is proved for finite $a$.

Let now $a$ be an arbitrary function that satisfies conditions of Theorem 2 ,

Let $g \in C_{0}^{\infty}(\mathbb{R}) ; g(x)=1,|x| \leq 1$. Put $g_{n}(x)=g(x / n), a_{n}(x)=g_{n}(x) a(x)$. Let $\varphi_{t}^{n}(x)$ be a solution of (11) with a drift coefficient equal to $a_{n}$.

Observe that by uniqueness of the solution we have the equality

$$
\varphi_{t}^{n}(x)=\varphi_{t}(x)
$$

for a.a. $\omega$ from the event $\left\{\sup _{z \in[0, t]}\left|\varphi_{z}(x)\right| \leq n\right\}$.

Let $[c, d]$ be an arbitrary interval. Denote $n_{0}=n_{0}(\omega)=\max _{z \in[0, t]}\left(\left|\varphi_{z}(c)\right|+\left|\varphi_{z}(d)\right|\right)$. Making use of (22), Proposition 1, and Fubini's theorem we obtain the equality $\varphi_{t}(x)=$ $\varphi_{t}^{n}(x)$ valid for all $n \geq n_{0}$, a.a. $\omega$, and $\lambda$-a.a. $x \in[c, d]$. Since $a_{n}$ is finite, $\varphi_{t}^{n}(\cdot) \in$ 
$W_{p}^{1}([c, d])$ a.s. Thus $\varphi_{t}(\cdot) \in W_{p}^{1}([c, d])$ a.s. and its derivatives coincide a.s. with that of $\varphi_{t}^{n}$ if $n \geq n_{0}$. The definition of the local time entails that $\alpha_{x}^{n}(y, t)=\alpha_{x}(y, t), n \geq n_{0}$, for $\lambda$-a.a. $y \in[c, d]$ with probability 1 . So formula (14) holds true.

Theorem 2 is proved.

\section{REFERENCES}

[1] O. V. Aryasova and A. Yu. Pilipenko. On properties of a flow generated by an sde with discontinuous drift. Electron. J. Probab., 17:no. 106, 1-20, 2012.

[2] S. Attanasio. Stochastic flows of diffeomorphisms for one-dimensional SDE with discontinuous drift. Electron. Commun. Probab., 15:no. 20, 213-226, 2010.

[3] S. M. Berman. Joint continuity of the local times of markov processes. Zeitschrift für Wahrscheinlichkeitstheorie und Verwandte Gebiete, 69:37-46, 1985.

[4] N. Bouleau and F. Hirsch. Dirichlet forms and analysis on Wiener space. De Gruyter studies in mathematics. W. de Gruyter, 1991.

[5] F. Flandoli, M. Gubinelli, and E. Priola. Flow of diffeomorphisms for SDEs with unbounded Hölder continuous drift. Bulletin des Sciences Mathematiques, 134(4):405 - 422, 2010.

[6] D. Geman and J. Horowitz. Occupation densities. Ann. Probab., 8:1-67, 1980.

[7] T. Komatsu. On the martingale problem for generators of stable processes with perturbations. Osaka J. Math, (21):113-132, 1984.

[8] H. Kunita. Stochastic Flows and Stochastic Differential Equations. Cambridge Univ. Press, 1990.

[9] A. Yu. Pilipenko. On existence and properties of strong solutions of one-dimensional stochastic equations with an additive noise. Theory of Stochastic Processes. (In print). arXiv:1306.0212v1 [math.PR].

[10] S. I. Podolynny and N. I. Portenko. On multidimensional stable processes with locally unbounded drift. Random Operators and Stoch. Equat., 3(2):113-124, 1995.

[11] N. I. Portenko. Non-negative additive functionals of a Markov process and some limit theorems (in Russian). Teor. Sluch. Protsess., (1):86-107, 1973.

[12] N. I. Portenko. Generalized diffusion processes. Translations of mathematical monographs. American Mathematical Society, 1990.

[13] N. I. Portenko. Some perturbations of drift-type for symmetric stable processes. Random Operators and Stoch. Equat., 2(3):211-224, 1994.

[14] C. Stricker and M. Yor. Calcul stochastique dépendant d'un paramètre. Z. Wahrscheinlichkeitstheor. Verw. Geb., 45:109-133, 1978.

Institute of Geophysics, National Academy of Sciences of Ukraine, Palladin Pr. 32, 03680, KIEV-142, UKRAINE

E-mail address: oaryasova@mail.ru

Institute of Mathematics, National Academy of Sciences of Ukraine, Tereshchenkivska STR. 3, 01601, KiEV, Ukraine

E-mail address: pilipenko.ay@yandex.ua 